\title{
Hypothermia and Fever After Organophosphorus Poisoning in Humans-A Prospective Case Series
}

\author{
Alison Moffatt • Fahim Mohammed • \\ Michael Eddleston • Shifa Azher • Peter Eyer • \\ Nick A. Buckley
}

Published online: 19 March 2010

(C) The Author(s) 2010. This article is published with open access at Springerlink.com

\begin{abstract}
There have been many animal studies on the effects of organophosphorus pesticide (OP) poisoning on thermoregulation with inconsistent results. There have been no prospective human studies. Our aim was to document the changes in body temperature with $\mathrm{OP}$ poisoning. A prospective study was conducted in a rural hospital in Polonnaruwa, Sri Lanka. We collected data on sequential patients with OP poisoning and analyzed 12 patients selected from 53 presentations who had overt signs and symptoms of OP poisoning and who had not received atropine prior to arrival. All patients subsequently received specific management with atropine and/ or pralidoxime and general supportive care. Tympanic temperature, ambient temperature, heart rate, and clinical examination and interventions were recorded prospectively throughout their hospitalization. Initial hypother-
\end{abstract}

Presented at the Asia Pacific Association of Medical Toxicology (APAMT) meeting, Bangkok, December 2007

A. Moffatt $\cdot$ F. Mohammed $\cdot$ M. Eddleston $\cdot$ S. Azher · P. Eyer $\cdot$

N. A. Buckley

South Asian Clinical Toxicology Research Collaboration,

Peradeniya, Sri Lanka

URL: www.sactrc.org

A. Moffatt

Clinical Pharmacology and Toxicology, Canberra Clinical School,

Canberra, ACT, Australia

F. Mohammed $\cdot$ M. Eddleston

Department of Clinical Medicine, Faculty of Medicine,

University of Colombo,

Colombo, Sri Lanka

\section{Eddleston}

Center for Tropical Medicine, Nuffield Department of Clinical

Medicine, University of Oxford,

Oxford, England, UK mia as low as $32^{\circ} \mathrm{C}$ was observed in untreated patients. Tympanic temperature increased over time from an early hypothermia $\left(<35^{\circ} \mathrm{C}\right.$ in $6 / 12$ patients) to later fever $(7 / 12$ patients $>38^{\circ} \mathrm{C}$ at some later point). While some of the late high temperatures occurred in the setting of marked tachycardia, it was also apparent that in some cases fever was not accompanied by tachycardia, making excessive atropine or severe infection an unlikely explanation for all the fevers. In humans, OP poisoning causes an initial hypothermia, and this is followed by a period of normal to high body temperature. Atropine and respiratory complications may contribute to fever but do not account for all cases.

Keywords Organophosphorus · Pesticide · Poisoning · Thermoregulation $\cdot$ Cholinergic

F. Mohammed $\cdot$ S. Azher

Polonnaruwa General Hospital,

Polonnaruwa, North Central Province, Sri Lanka

P. Eyer

Walther-Straub-Institute of Pharmacology and Toxicology, Ludwig Maximilians University of Munich, Munich 80336, Germany

\section{N. A. Buckley $(\square)$}

Prince of Wales Hospital Clinical School, University of NSW, Level 1, South Wing Edmund Blackett Building,

Randwick, New South Wales 2031, Australia

e-mail: n.buckley@unsw.edu.au 


\section{Introduction}

Organophosphorus pesticide (OP) self-poisoning is a significant cause of morbidity and mortality in the developing world [1-3], with the in-hospital case fatality usually reported to be between $10 \%$ and $20 \%$ [1]. OPs are acetylcholinesterase (AChE) inhibitors, thereby inhibiting the metabolism of acetylcholine (ACh). The rapid accumulation of $\mathrm{ACh}$ in the synaptic junctions of central nervous system and peripheral tissues results in a "cholinergic crisis," characterized by receptor overstimulation and a range of muscarinic, nicotinic, and central effects [4]. The mainstay of treatment involves atropine, a central and peripheral muscarinic receptor antagonist, and pralidoxime chloride, which reactivates inhibited AChE [5].

However, the understanding of the pathophysiology of OP poisoning is incomplete in many respects. OPs have been reported to interfere with the control of ACh-regulated homeostatic mechanisms such as temperature regulation [6]. The current data on cholinergic influences on temperature regulation are largely derived from animal studies. Reports of direct CNS administration of cholinergic agonists in the region of the hypothalamus or cerebral ventricles of laboratory rodents resulting in hypothermia are in contrast with other studies reporting just the opposite, that is, heat production and/or conservation [6,7]. This may be related to dose; generally higher doses were associated with hypothermia, and hyperthermia was only seen with lower doses [6]. Other studies have demonstrated that OP poisoning results in a period of hypothermia followed by a fever of delayed onset [8-11], without exhibiting any of the other classical signs of bacterial or viral infection. Further complicating the literature, hot and cold environments have been found to modify the sensitivity of rats and primates to $\mathrm{AChE}$ inhibitors and also hypothermic and hyperthermic responses in animals $[6,12]$.

It is not clear whether these observations apply to acute human poisoning. Review of the literature regarding human responses to OP poisoning appears to suggest that an elevation in body temperature is a frequent outcome, but cases are complicated by concurrent illnesses (in particular aspiration pneumonitis/pneumonia) and interventions that may of themselves produce high temperatures, in particular anticholinergic agents [13-16]. In these cases, it is unclear whether atropine was responsible for the temperature or whether high doses of atropine were given to patients with severe OP poisoning, and fever was a manifestation of severe OP poisoning. Most reports of human cases with fever following OP ingestion come from the warmer regions of the world, such as South Asia [1-3, 17, 18], the Middle East [13], and Africa [19]. Therefore, reported fever in patients with OP poisoning may represent a loss of normal thermoregulation.
The human data on OP poisoning are from case studies based on records of routinely recorded vital signs. The aim of this study was to prospectively and closely observe a series of patients admitted to a regional hospital in Sri Lanka with acute OP poisoning and document the changes in their body temperature throughout the length of their hospital stay.

\section{Methods}

A prospective study into the effects of OP poisoning on tympanic temperature (as an indicator of core body temperature) was undertaken at Polonnaruwa General Hospital $(\mathrm{PGH})$ in the agricultural region of North Central Sri Lanka. It formed part of an established study into the management of acute self-poisoning approved by the Colombo Faculty of Medicine Ethics Committee, the Oxfordshire Clinical Research Ethics Committee, and the Australian National University HREC Protocol 2003/227.

All patients admitted directly or transferred to PGH with a history of self-poisoning with an OP pesticide were included in an initial study period of 20 days. It became apparent during this time that treatment received in the initial resuscitation may be having a marked effect on the recorded temperature. Therefore, a further study period of 98 days followed, in which patients with a history of OP poisoning were only included if they had overt signs and symptoms of OP poisoning and yet had not received atropine prior to arrival at PGH. We present data from both periods only on those that presented meeting these criteria.

Each patient was seen on admission, and data were recorded prospectively. The poison ingested was identified from the patients' or relatives' histories, the bottle brought with the patient, or the doctor's comments in transfer letters. Consent was gained from all study participants. Tympanic temperature was measured as frequently as was possible (without interfering with medical care) during the first $6 \mathrm{~h}$ of admission and approximately 3-hourly thereafter. All temperatures were taken with the same Braun ThermoScan digital ear thermometer, and medical staff received basic training in the technique required for best accuracy [20]. The ambient temperature was recorded simultaneously with a mercury thermometer. Other relevant clinical information including symptoms and signs of poisoning and antidote administration were recorded, with further management and patient progress details obtained from a review of patient notes. Most patients were managed on open wards, which are not air-conditioned and have few fans. The ambient temperature typically varied somewhere between $24^{\circ} \mathrm{C}$ and $35^{\circ} \mathrm{C}$ on these wards. Some patients were managed in $\mathrm{PGH}$ intensive care which is airconditioned to a temperature of around $25^{\circ} \mathrm{C}$. 
Each patient was treated in accordance with a standard management protocol [21]. All patients received specific management with titrated doses of atropine and/or pralidoxime chloride ( $1 \mathrm{~g}$ 6-hourly for around $48 \mathrm{~h}$ ), and all patients received ongoing general supportive care.

Body temperature, ambient temperature, and heart rate were initially plotted against time using GraphPad Prism [version 3.02 for Windows (GraphPad Software, San Diego, CA, USA, www.graphpad.com)]. Significant clinical notes, the time of the first temperature measurements, and the normal range for core body temperature (as defined by Vassallo and Delaney 1998 [22]) were added at the corresponding points on the graphs.

Where possible, admission plasma samples were taken for butyrylcholinesterase activity (to show exposure) and pesticide concentration. Plasma was separated and frozen at $-20^{\circ} \mathrm{C}$ until analysis. All analyses were done at Peter Eyer's laboratory in Munich, Germany. Butyrylcholinesterase activity was assessed as described previously [23]. Concentrations of organophosphorus pesticides in plasma were quantified by reversed-phase high-performance liquid chromatography and ultraviolet detection. The lower limits of quantitation were $1 \mu \mathrm{mol} / \mathrm{l}$ plasma for dimethoate and $0.1 \mu \mathrm{mol} / \mathrm{l}$ plasma for chlorpyrifos and quinalphos.

\section{Results}

Over the two study periods, 53 patients were admitted to PGH with deliberate OP self- poisoning. Of these patients, 41 were excluded because they were initially asymptomatic (17) or had received atropine therapy prior to admission (24). There were 11 male patients and one female patient, with a mean age of 37.9 years (range 17-65), and they had ingested a range of OP (Table 1). All 12 patients demonstrated manifestations of OP activity at muscarinic and/or nicotinic receptors and had presented within $6 \mathrm{~h}$ of ingestion. Five patients had a reduced level of consciousness on admission. Seven required intubation and admission to the intensive care unit (ICU). One of these seven whose admission was complicated by aspiration pneumonia died unexpectedly 8 days after admission, although he had been apparently stable following extubation the preceding day.

To demonstrate the data collected, Fig. 1 shows the temperature readings for the patient with the greatest disturbance of temperature. This was a 43-year-old male admitted three and a half hours after ingestion of unknown quantities of chlorpyrifos and alcohol. He received only IV saline prior to transfer and on arrival had a Glasgow Coma Scale (GCS) of 4/15, pinpoint pupils (1 mm), vomiting, and diarrhea, was diaphoretic, and had bilateral lung crepitations. Pulse was $72 \mathrm{bpm}$ and BP 140/80. The patient was immediately intubated and given a total of $4 \mathrm{mg}$ atropine in bolus doses. An atropine infusion of $1.2 \mathrm{mg} / \mathrm{h}$ was commenced $1 \mathrm{~h}$ later and continued for approximately $6 \mathrm{~h}$. Seven hours after admission, the patient was transferred to the ICU where he was paralyzed and sedated with atracurium and morphine, respectively, and given pralidoxime chloride $1 \mathrm{~g} \mathrm{q} 6 \mathrm{~h}$. He remained in a similar state for the following 3 days, after which no more data were recorded. The patient was later discharged.

The figure demonstrates severe hypothermia $\left(32.2^{\circ} \mathrm{C}\right)$ on admission, coinciding with classical signs of OP poisoning. A rise in tympanic temperature was observed to follow administration of atropine and hypothermia resolved by $12 \mathrm{~h}$ postingestion (Fig. 1). Thereafter, in the relatively stable temperature of the ICU, the patient's temperature remained within the normal range, except for the final measurement, which was slightly below normal.

The tympanic temperatures and heart rates of individual patients with respect to time post-OP ingestion are shown in Fig. 2. The same pattern is seen when the two patients with normal butyrylcholinesterase activity are removed (data not shown). A trend of tympanic temperature increasing from an early hypothermia to later mild fever is demonstrated. While some of the late high temperatures occurred in the setting of marked tachycardia, it is also apparent that in some cases fever was not accompanied by tachycardia, making excessive atropine an unlikely explanation for all the fevers. There was no visual relationship observed between ambient and core temperature in any of these patients nor was there an apparent relationship between initial temperature and ambient temperature (data not shown).

\section{Discussion}

This study was the first attempt to systematically and prospectively record the tympanic temperatures of patients admitted to hospital with acute OP self-poisoning. We have confirmed that alterations in body temperature and thermoregulation are common in symptomatic OP poisoning, but there is a great deal of variation largely due to treatment and complications. We have identified a clear pattern of early hypothermia in significant poisoning which corrects with initial treatment with somewhat variable and smaller effects thereafter that are less easily attributed to specific factors frequently leading to above normal temperatures a few days later.

These results contrast those of numerous animal studies purporting purely hypothermic or hyperthermic responses to OP and those that suggest that there is a significant correlation between OP exposure and hypersensitivity to ambient temperatures $[12,24]$. The data from this study 
Table 1 Patients admitted to Polonnaruwa General Hospital with acute OP poisoning who were symptomatic and had received no prior atropine therapy

\begin{tabular}{|c|c|c|c|c|c|c|c|c|c|c|c|c|}
\hline Study number & 1427 & 1430 & 1436 & 1447 & 2126 & 2180 & 2169 & 2161 & 2116 & 2342 & 2142 & 2196 \\
\hline Male & $\checkmark$ & $\checkmark$ & $\checkmark$ & $\checkmark$ & & $\checkmark$ & $\checkmark$ & $\checkmark$ & $\checkmark$ & $\checkmark$ & $\checkmark$ & $\checkmark$ \\
\hline Age & 17 & 34 & 32 & 43 & 22 & 32 & 40 & 50 & 59 & 26 & 35 & 65 \\
\hline OP ingested & $\mathrm{CP}$ & $\mathrm{CP}$ & UO & $\mathrm{CP}$ & QP & QP & $\mathrm{DZ}$ & $\mathrm{DM}$ & $\mathrm{DM}$ & $\mathrm{DM} / \mathrm{CP}$ & $\mathrm{DM}$ & $\mathrm{DM}$ \\
\hline Ethanol coingestion & & $\checkmark$ & $\checkmark$ & $\checkmark$ & & & & & & & & \\
\hline GCS on arrival (out of 15 ) & 15 & 15 & 14 & 4 & 15 & 15 & 15 & 15 & 3 & 7 & 4 & 15 \\
\hline Time to admission (h) & 3 & 0.5 & 5.5 & 3.5 & 6 & 3 & 4.5 & 3 & 3 & 1 & 4 & 1 \\
\hline Admission temperature & 36.2 & 35.9 & 35.3 & 32.2 & 34.3 & 36.0 & 37.4 & 35.7 & 35.4 & 37.2 & 36.4 & 36.8 \\
\hline Time to lowest temperature (h) & 11.2 & 1.5 & 5.6 & 3.7 & 4.4 & 10.7 & 18 & 5.4 & 10.6 & 2.0 & 4.7 & 6.2 \\
\hline Lowest recorded temperature & 35.4 & 35.1 & 35.1 & 32.2 & 34.0 & 35.9 & 36.9 & 34.5 & 34.8 & 34.7 & 36.4 & 34.7 \\
\hline Time to highest temperature (h) & 51 & 48 & 24 & 17.3 & 12.4 & 158 & 6.8 & 380 & 116 & 65 & 177 & 48 \\
\hline Highest recorded temperature & 37.1 & 37.9 & 37.0 & 37.5 & 38.0 & 38.6 & 38.2 & 39.7 & 39.4 & 37.3 & 38.8 & 38.5 \\
\hline Admission $\mathrm{BChE}$ & 251 & 50 & $\mathrm{NC}$ & 22 & 10 & 3,338 & 3,989 & 1,481 & 1,431 & 10 & 60 & 2,067 \\
\hline OP concentration $(\mu \mathrm{M})$ & 0.29 & 4.3 & $\mathrm{NC}$ & NA & 4.68 & NA & NA & 323 & 66 & $25 / 1.2$ & 8 & 432 \\
\hline ICU admission/intubation & & & & $\checkmark$ & $\checkmark$ & & $\checkmark$ & $\checkmark$ & $\checkmark$ & $\checkmark$ & $\checkmark$ & \\
\hline Outcome & $\mathrm{D} / \mathrm{C}$ & $\mathrm{D} / \mathrm{C}$ & $\mathrm{D} / \mathrm{C}$ & $\mathrm{D} / \mathrm{C}$ & $\mathrm{D} / \mathrm{C}$ & $\mathrm{D} / \mathrm{C}$ & $\mathrm{D} / \mathrm{C}$ & $\mathrm{D} / \mathrm{C}$ & RIP & $\mathrm{D} / \mathrm{C}$ & $\mathrm{D} / \mathrm{C}$ & $\mathrm{D} / \mathrm{C}$ \\
\hline
\end{tabular}

$D / C$ discharged, $C P$ chlorpyrifos, $R I P$ deceased, $D M$ dimethoate, $Q P$ Quinalphos, $D Z$ Diazinon, $U O$ unknown organophosphate, $B C h E$ butyrylcholinesterase activity (normal range 3,200-5,200 mU/ml [23]), NC not collected, $N A$ not analyzed

suggest that, in humans receiving antidotes, an initial hypothermic response is followed by a delayed fever. This relationship has been suggested in a small number of animal studies [8-11].
On the graphs, hypothermia is shown as a lowering of the tympanic body temperature to less than or equal to $35^{\circ} \mathrm{C}$, based on generally accepted values in clinical medicine [22]. However, it has been noted that core
Fig. 1 Heart rate, tympanic temperature, and ambient temperature following ingestion of OP (pt. 1447)

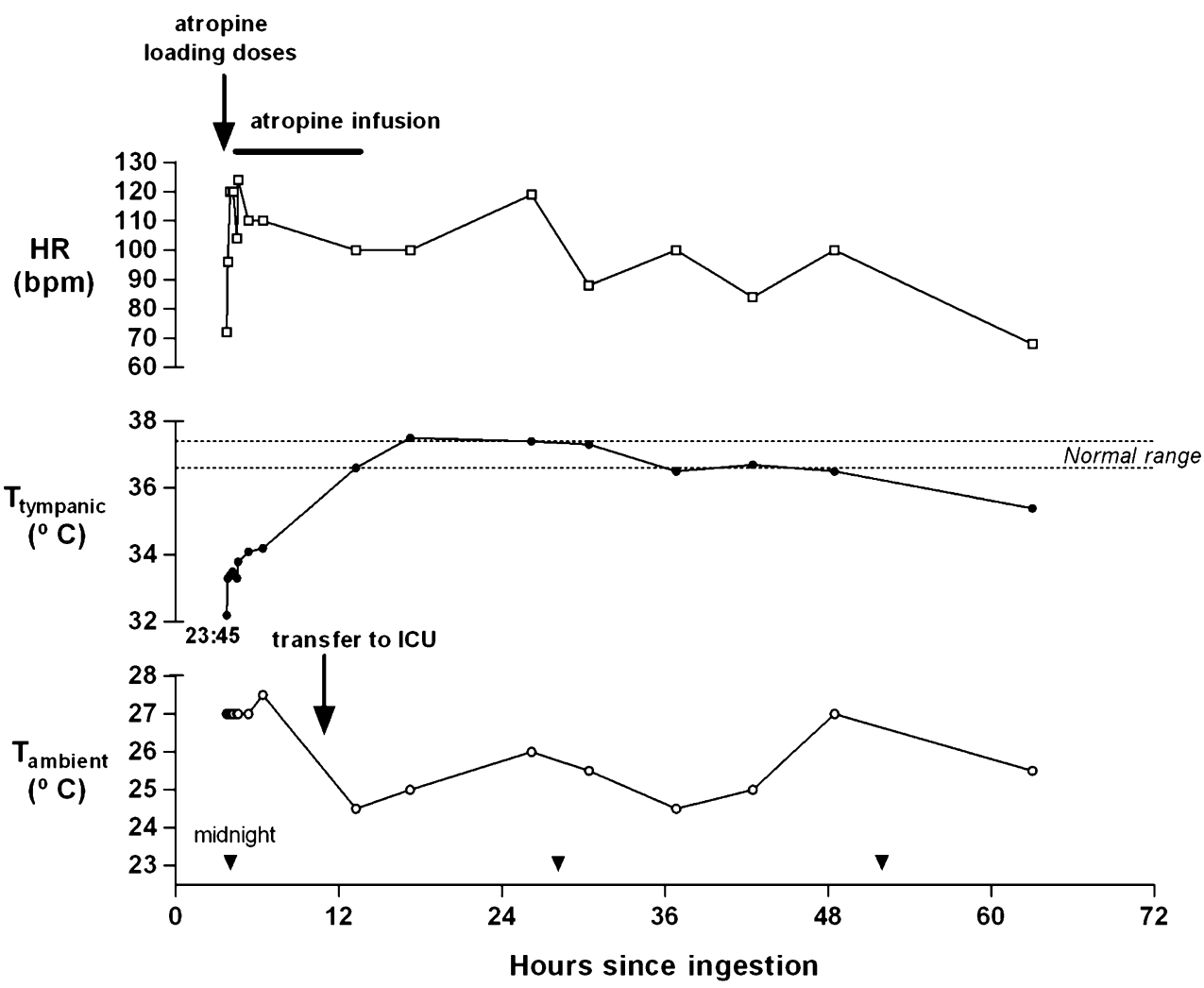


Fig. 2 The tympanic temperatures and heart rates of individual subjects in Table 1 against time since OP ingestion, from admission until discharge from hospital. Note that temperatures are consistently lownormal early and become normal or high after $24-48 \mathrm{~h}$, while heart rate is evenly scattered and shows little change over time, indicating atropine therapy and infection (which would both cause tachycardia as well as a temperature rise) are not likely to be responsible for the trends. Two patients $(x+)$ appear to have much less severe poisoning as $\mathrm{BChE}$ was not depressed. Hyperbolic equation for other ten: Temp $=35.4+$ $2.8 \times$ hours $/($ hours +25$)$
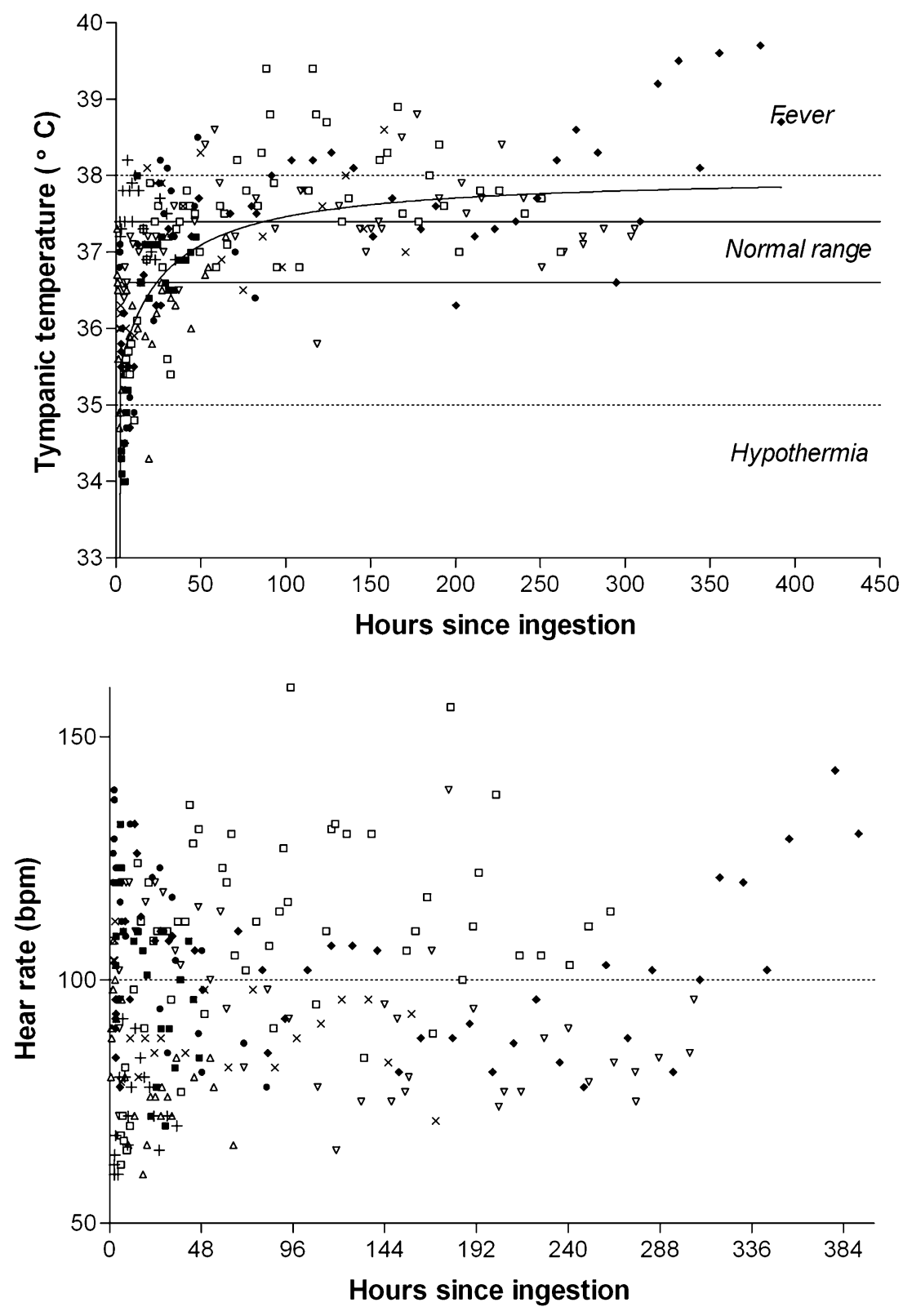

temperature does not normally reach even $36^{\circ} \mathrm{C}$ without triggering behavioral and autonomic cold defenses, suggesting that physiological hypothermia would be closer to $36^{\circ} \mathrm{C}$ [25]. Using this cutoff, six of the 12 patients had hypothermia on admission and 11 within the first $12 \mathrm{~h}$.

While most publications describe fluctuations in body temperature noticed retrospectively from examination of patient notes, we attempted to record temperatures prospectively at very frequent intervals while recording other factors that might influence temperature. However, there were still several limitations to the accurate determination of the impact of OP poisoning on thermoregulation in humans. The most important is the need to treat patients with antidotes that also may affect temperature regulation. Moreover, the treatment is individualized to each patient
Table 2 Clinical notes coinciding with a rise in body temperature in patients with OP poisoning at PGH

Drug administration

Atropine (most common factor)

Chlorpromazine

Diazepam

Carbamazepine

Benzhexol

Signs of alcohol withdrawal

Use of physical restraint

Lower respiratory tract infection 
depending on, for example, whether they had seizures, were aggressive, or were developing a respiratory tract infection. Table 2 lists some of the many factors known to alter thermoregulation that we noted in the first 20 days may have influenced patient temperatures in this study. It would have been useful to have more facilities to exclude infection in those with late onset of fevers. Body temperatures may also have been influenced by a patient's location. That is, whether they were near an electric fan, in the center of an overcrowded ward, or whether they were in the more controlled environment of the intensive care unit. However, ambient temperature did not seem to have an obvious relationship with tympanic temperature in most patients.

Another unavoidable limitation was the timing of the start for recording thermometry. While one patient presented to PGH $30 \mathrm{~min}$ after OP ingestion, another was admitted $6 \mathrm{~h}$ postingestion. Finally, the patients in this study may not be representative of all patients presenting with OP poisoning. Although the number of patients included in this study was small, there was only one death due to OP poisoning, which is less than the estimated $15 \%$ to $20 \%$ mortality rate for symptomatic OP poisoning [1]. The doses of poison ingested are unknown, meaning data analysis from a dose-response perspective is impossible.

It is possible that the temperature aberration in some patients was due to alcohol, although the three patients with known ingestion of ethanol were not clearly different from the rest (Table 1). Effects of ethanol in humans at the ambient temperatures in Sri Lanka have been neither consistent nor significant [26]. It is also possible (but unlikely) that effects on temperature in OP poisoning patients are due to solvents rather than the OP.

Future studies may be able to explore the mechanism for heat dissipation in early organophosphorus poisoning, for example by measuring skin blood flow and temperature and the differences between core and peripheral temperature. Such studies might also address the issue of whether hyperthermia contributes to later elevated temperatures. More studies could also be done exploring the medium to long-term effects on thermoregulation after the acute poisoning period. OP are well known to produce long-lasting effects in humans in the form of an intermediate syndrome (impaired neuromuscular transmission 24 to $96 \mathrm{~h}$ after exposure) and delayed polyneuropathy, which may develop days to weeks after the exposure [4]. Regular temperature measurement in patients experiencing these delayed sets of symptoms may also show a pattern of temperature changes that might be attributed to the action of OP on thermoregulation.

\section{Conclusions}

This study has allowed a generalization to be made that, in humans, OP poisoning causes an initial fall in body temperature, and this is followed by a period of normal to high body temperature, as measured by tympanic thermometry. Of the many possible confounders, it is the life-saving medical interventions received by each patient and frequent respiratory complications that are the greatest hindrances to the accurate observation of trends in body temperature during the period of intensive management following admission to hospital. Thus, the level of experimental control required for a more conclusive answer on the direct acute effects of OP poisoning in humans is unattainable.

Acknowledgements/Funding We thank the doctors of the SACTRC-OxCol Poisoning Study Team and their patients at Polonnaruwa General Hospital for their assistance in conducting this study. ME is a Wellcome Trust Career Development Fellow, funded by grant GR063560. The South Asian Clinical Toxicology Research Collaboration is funded by a Wellcome Trust/National Health and Medical Research Council International Collaborative Research Grant GR071669. The authors declare that they have no competing interests.

Open Access This article is distributed under the terms of the Creative Commons Attribution Noncommercial License which permits any noncommercial use, distribution, and reproduction in any medium, provided the original author(s) and source are credited.

\section{References}

1. Eddleston M (2000) Patterns and problems of deliberate selfpoisoning in the developing world. QJM 93:715-731

2. Eddleston M, Konradsen F (2007) Commentary: time for a reassessment of the incidence of intentional and unintentional injury in India and South East Asia. Int J Epidemiol 36:208-211

3. Gunnell D, Fernando R, Hewagama M, Priyangika W, Eddleston M (2007) The impact of pesticide regulations on suicide in Sri Lanka. Int J Epidemiol 36:1235-1242

4. Johnson MK, Jacobsen D, Meredith TJ, Eyer P, Heath AJ, Ligtenstein DA et al (2000) Evaluation of antidotes for poisoning by organophosphorus pesticides. Emerg Med 12:22-37

5. Eddleston M, Buckley NA, Eyer P, Dawson AH (2008) Management of acute organophosphorus pesticide poisoning. Lancet 371:597-607

6. Gordon CJ (1994) Thermoregulation in laboratory mammals and humans exposed to anticholinesterase agents. Neurotoxicol Teratol $16: 427-453$

7. Gordon CJ (1995) Factors influencing diisopropyl fluorophosphateinduced hypothermia and hyperthermia in the rat. Neurotoxicol Teratol 17:679-683

8. Gordon CJ, Fogelson L, Richards J, Highfill J (1991) Relationship between cholinesterase inhibition and thermoregulation following exposure to diisopropyl fluorophosphate in the rat. Toxicol Lett 59:161-168

9. Gordon CJ, Grantham TA, Yang Y (1997) Hypothermia and delayed fever in the male and female rat exposed to chlorpyrifos. Toxicology 118:149-158 
10. Gordon CJ, Mohler FS, Watkinson WP, Rezvani AH (1988) Temperature regulation in laboratory mammals following acute toxic insult. Toxicology 53:161-178

11. Johnson RP, Gordon CJ (1997) Tolerance to the hypothermic and hyperthermic effects of chlorpyrifos. Toxicology 121:215-221

12. Gordon CJ (1997) Behavioral thermoregulatory response to chlorpyrifos in the rat. Toxicology 124:165-171

13. Saadeh AM, al-Ali MK, Farsakh NA, Ghani MA (1996) Clinical and sociodemographic features of acute carbamate and organophosphate poisoning: a study of 70 adult patients in north Jordan. $\mathrm{J}$ Toxicol Clin Toxicol 34:45-51

14. Ochi G, Watanabe K, Tokuoka H, Hatakenaka S, Arai T (1995) Neuroleptic malignant-like syndrome: a complication of acute organophosphate poisoning. Can J Anaesth 42:1027-1030

15. Hantson P, Hainaut P, Vander SM, Mahieu P (1996) Regulation of body temperature after acute organophosphate poisoning. Can $\mathrm{J}$ Anaesth 43:755

16. Namba T, Nolte CT, Jackrel J, Grob D (1971) Poisoning due to organophosphate insecticides. Acute and chronic manifestations. Am J Med 50:475-492

17. Karalliedde L, Senanayake N (1988) Acute organophosphorus insecticide poisoning in Sri Lanka. Forensic Sci Int 36:97-100

18. Agarwal SB (1993) A clinical, biochemical, neurobehavioural, and sociopsychological study of 190 patients admitted to hospital as a result of acute organophosphate poisoning. Environ Res $62: 63-70$
19. Nhachi CF (1988) An evaluation of organophosphate poisoning cases in an urban setting in Zimbabwe. East Afr Med J 65:588-592

20. Chamberlain JM, Terndrup TE, Alexander DT, Silverstone FA, Wolf-Klein G, O'Donnell R et al (1995) Determination of normal ear temperature with an infrared emission detection thermometer. Ann Emerg Med 25:15-20

21. Eddleston M, Dawson A, Karalliedde L, Dissanayake W, Hittarage A, Azher S et al (2004) Early management after selfpoisoning with an organophosphorus or carbamate pesticide-a treatment protocol for junior doctors. Crit Care 8:R391-R397

22. Vassallo S, Delaney KA (1998) Thermoregulatory principles. In: Goldfrank L, Flomenbaum N, Lewin NA, Weisman R, Howland MA, Hoffman RS (eds) Goldfrank's toxicologic emergencies. Appleton \& Lange, Stamford, pp 285-307

23. Eddleston M, Eyer P, Worek F, Rezvi Sheriff MH, Buckley NA (2008) Predicting outcome using butyrylcholinesterase activity in organophosphorus pesticide self-poisoning. QJM 101:467-474

24. Gordon CJ (1994) Thermoregulatory effects of chlorpyrifos in the rat: long-term changes in cholinergic and noradrenergic sensitivity. Neurotoxicol Teratol 16:1-9

25. Sessler D (1998) Poikilothermia in man: pathophysiological aspects and clinical implications (book review). Anesthesiology $88: 841-842$

26. Kalant H, Le AD (1983) Effects of ethanol on thermoregulation. Pharmacol Ther 23:313-364 